University of Wollongong

Research Online

Australian Institute for Innovative Materials -

Papers

Australian Institute for Innovative Materials

$1-1-2013$

\title{
Effect of polymer ligand structures on fluorescence of gold clusters prepared by photoreduction
}

Luo Li

Huazhong University of Science and Technology

Zhen Li

University of Wollongong, zhenl@uow.edu.au

Hui Zhang

Huazhong University of Science and Technology

Shoucun Zhang

Huazhong University of Science and Technology

Irfan Majeed

Huazhong University of Science and Technology

See next page for additional authors

Follow this and additional works at: https://ro.uow.edu.au/aiimpapers

Part of the Engineering Commons, and the Physical Sciences and Mathematics Commons

Research Online is the open access institutional repository for the University of Wollongong. For further information contact the UOW Library: research-pubs@uow.edu.au 


\title{
Effect of polymer ligand structures on fluorescence of gold clusters prepared by photoreduction
}

\author{
Abstract \\ Blue emission fluorescent Au5 clusters with maximum quantum yield of $20.1 \%$ were synthesized by a \\ simple photoreduction method using three specially designed tridentate polymer ligands. The evolution of \\ fluorescent Au nanoclusters (Au NCs) under UV irradiation was studied by fluorescence, UV-Vis and X-ray \\ photoelectron spectroscopic techniques, suggesting that the fluorescence of Au NCs is size-dependent \\ and is associated with the presence of $\mathrm{Au}(\mathrm{i})$ ions in the Au NCs. The effect of polymer structure on the \\ fluorescent Au NCs has also been discussed. These highly fluorescent Au NCs have potential \\ applications in the fabrication of optoelectronic devices and light emitting materials.
}

\section{Keywords}

fluorescence, gold, clusters, prepared, photoreduction, effect, polymer, ligand, structures

Disciplines

Engineering | Physical Sciences and Mathematics

\section{Publication Details}

Li, L., Li, Z., Zhang, H., Zhang, S., Majeed, I. \& Tan, B. (2013). Effect of polymer ligand structures on fluorescence of gold clusters prepared by photoreduction. Nanoscale, 5 (5), 1986-1992.

\section{Authors}

Luo Li, Zhen Li, Hui Zhang, Shoucun Zhang, Irfan Majeed, and Bien Tan 


\title{
Nanoscale
}

\section{PAPER}

Cite this: Nanoscale, 2013, 5, 1986

\section{Effect of polymer ligand structures on fluorescence of gold clusters prepared by photoreductiont}

\author{
Luo Li, ${ }^{\text {a }}$ Zhen Li, ${ }^{\text {b }}$ Hui Zhang, ${ }^{a}$ Shoucun Zhang, ${ }^{a}$ Irfan Majeed ${ }^{\text {a }}$ and Bien Tan ${ }^{* a}$ \\ Blue emission fluorescent $\mathrm{Au}_{5}$ clusters with maximum quantum yield of $20.1 \%$ were synthesized by a \\ simple photoreduction method using three specially designed tridentate polymer ligands. The evolution \\ of fluorescent Au nanoclusters (Au NCs) under UV irradiation was studied by fluorescence, UV-Vis and \\ $\mathrm{X}$-ray photoelectron spectroscopic techniques, suggesting that the fluorescence of Au NCs is size- \\ dependent and is associated with the presence of $\mathrm{Au}(\mathrm{I})$ ions in the Au NCs. The effect of polymer \\ structure on the fluorescent Au NCs has also been discussed. These highly fluorescent Au NCs have \\ potential applications in the fabrication of optoelectronic devices and light emitting materials.
}

Received 18th November 2012 Accepted 29th December 2012

DOI: $10.1039 / \mathrm{c} 2 \mathrm{nr} 33693 f$

www.rsc.org/nanoscale

Among the metal nanoclusters, Au nanoclusters (Au NCs), which are less toxic, more biocompatible, and highly stable under ambient conditions, have attracted more attention for potential applications than other noble metal materials. ${ }^{30}$ In our previous work, $^{15}$ PTMP-PVAc polymer ligand was employed to synthesize fluorescent Au NCs. However, the fluorescence of PTMP-PVAc itself makes it difficult to ascertain whether the fluorescence comes from the Au NCs. In this paper, three types of non-fluorescent tridentate thioetherterminated polymer ligands, poly(methyl methacrylate) (PTMP-PMMA), poly(n-butyl methacrylate) (PTMP-PBMA) and poly(tert-butyl methacrylate) (PTMP-P $t$ BMA) were designed to synthesize blue fluorescent Au NCs by a simple and facile photoreduction method (Scheme 1). The highest fluorescence quantum yields (QY) of Au NCs stabilized with these nonfluorescent ligands PTMP-PMMA, PTMP-PBMA and PTMPPtBMA were found to be $3.8,14.3$ and $20.1 \%$, respectively, which are much higher than that of red emission Au NCs prepared in our previous work. ${ }^{8,25}$ The use of non-fluorescent ligands clearly proved the observed fluorescence originates from the Au NCs rather than the ligands themselves. Moreover, these designed ligands are very useful for studying the role of polymer structure in the formation of fluorescent $\mathrm{Au}$ NCs. Compared to other methods, ${ }^{31-34}$ the photoreduction approach is simple and feasible for avoiding the use of additional reducing agents, which is good for studying the photofluorescence mechanism of Au NCs. These multidentate polymer ligands have better protective capability than monodentate polymer ligands. ${ }^{35-37}$ Comparing to the bio-imaging applications of water-soluble red-emitting Au NCs prepared in our previous work, ${ }^{8,25}$ the resultant blue-emitting $\mathrm{Au}_{5}$ clusters are also equally important because of their applications in the field of light emitting materials and optoelectronic devices for their high QY. 


\author{
(a)
}

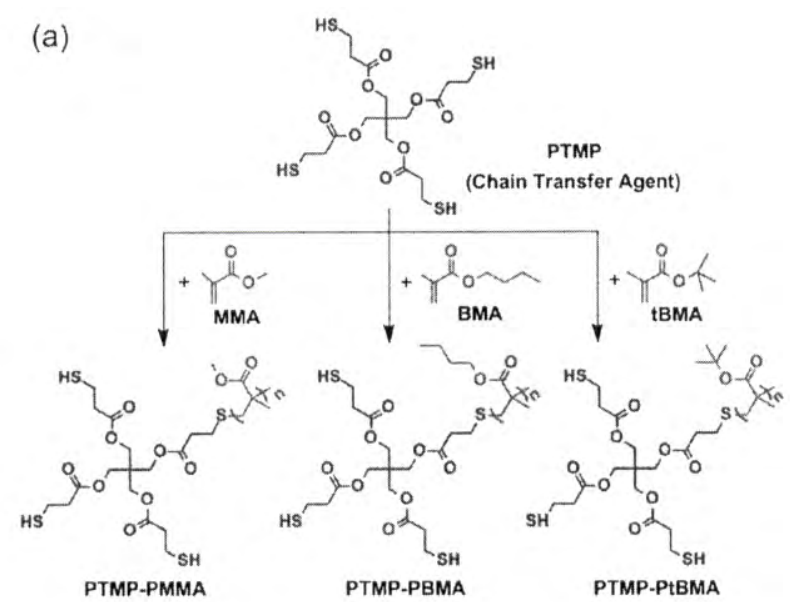

(b)

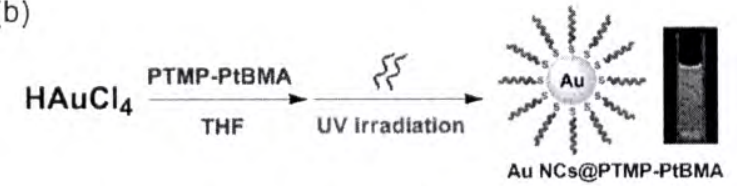

Scheme 1 The reaction scheme for (a) synthesis of tridentate polymer ligands PTMP-PMMA, PTMP-PBMA and PTMP-PtBMA. The repeating units number $(n)$ for PTMP-PMMA, PTMP-PBMA and PTMP-PtBMA were 38, 36 and 37, respectively, calculated by NMR. (b) Preparation of fluorescent Au NCs stabilized by tridentate polymer ligands in a homogeneous organic system.

\section{Experimental}

\subsection{Chemicals}

All chemicals are of analytical grade and used as received without any further purification, unless otherwise described. Methyl methacrylate (MMA, 99\%), $n$-butyl methacrylate ( $n$ BMA, $99 \%)$, tert-butyl methacrylate ( $t$ BMA, 99\%), hydrogen tetrachloroaurate $\left(\mathrm{HAuCl}_{4} \cdot 4 \mathrm{H}_{2} \mathrm{O}, 47.6 \%\right), 2,2^{\prime}$-azobisisobutyronitrile (AIBN), anhydrous ethanol, methanol, tetrahydrofuran (THF) and 1,4-dioxane were purchased from National Medicines Corporation Ltd of China. Pentaerythritol tetrakis-3-mercaptopropionate (PTMP, 97\%) was obtained from Aldrich.

\subsection{Synthesis of polymer ligands}

The synthesis of PTMP-PtBMA is described here as an example of preparation of polymer ligands. In a typical synthesis, $40 \mathrm{~mL}$ 1,4-dioxane solution containing monomer $t$ BMA $(7.110 \mathrm{~g}, 50$ mmol), chain-transfer agent PTMP (0.489 g, $1.00 \mathrm{mmol})$ and initiator AIBN $(0.082 \mathrm{~g}, 0.50 \mathrm{mmol})$ were added to a roundbottom flask fitted with a reflux condenser under nitrogen protection with magnetic stirring. The temperature of the reaction mixture was maintained at $80^{\circ} \mathrm{C}$ and refluxed for $6 \mathrm{~h}$. The viscous products were isolated by precipitating into a cold mixture of methanol and water (volume ratio $3: 1$ ). A fraction of low molar mass polymer, unreacted monomer and some oligomers formed due to incomplete reaction were removed during the precipitation step. The excess solvent was removed by evaporation using a vacuum oven set at $40{ }^{\circ} \mathrm{C}$ for $24 \mathrm{~h}$, and the yield was 91\%. PTMP-PMMA and PTMP-PBMA were synthesized by using MMA and BMA as monomers in a similar way; their yields were 82 and $80 \%$, respectively. The molecular weights of these three polymers were calculated based on the ratio of monomer units to the terminal group in the ${ }^{1} \mathrm{H}$ NMR spectra. The polymer molecular weights were further determined with gel permeation chromatography (GPC) by using polystyrene as calibration standard. Comparison of molecular weights measured by NMR and GPC are listed in Table S1 (ESI + ).

\subsection{Preparation of Au nanoclusters}

$\mathrm{Au}$ NCs were prepared according to the following method. Polymer ligand (PTMP-PMMA, PTMP-PBMA or PTMP-P $t$ BMA) was added into $5 \mathrm{~mL}$ of $1 \mathrm{mM}$ THF solution of $\mathrm{HAuCl}_{4}$ and incubated for $30 \mathrm{~min}$, the final concentration of polymer ligand was set to $4 \mathrm{mM}$, then subjected to an ultraviolet light source $(8 \mathrm{~W}$, wavelength $=365 \mathrm{~nm})$ for various time intervals. The asprepared Au NCs solution was first centrifuged at $16000 \mathrm{rpm}$ two times and then purified using a microporous filter $(0.22 \mu \mathrm{m})$. The remaining steps followed our previous work. ${ }^{15}$

\subsection{Characterization}

Fluorescence excitation and emission spectra were obtained by a FP-6500 fluorescence spectrometer (Jasco, JPN). UV-Vis absorption spectra were determined using a UV2550 Spectrophotometer (SHIMADZU, JPN). Dynamic light scattering (DLS) measurements were carried out with a ZetaPlus (Brookhaven, USA), which has an effective detection capability from 0.6 to 6000 $\mathrm{nm}$. TEM images were recorded with a Tecnai G2 electron microscope (FEI, USA) at an acceleration voltage of $200 \mathrm{kV}$. A drop of Au NCs solution was placed on carbon film coated copper grids. X-Ray photoelectron spectroscopy (XPS) spectra were measured with an AXIS-ULTRA DLD (Shimadzu, Japan). The energy resolution was set to $1.7 \mathrm{eV}$ to minimize data. Matrixassisted laser desorption ionization time-of-flight (MALDI-TOF) was analyzed on a Bruker Autoflex MALDI-TOF instrument. Samples $(0.5 \mu \mathrm{L})$ were spotted on a MALDI target plate with $0.5 \mu \mathrm{L}$ CHCA matrix (10 $\mathrm{mg} \mathrm{mL}^{-1} \mathrm{CHCA}$ in $50 \% \mathrm{ACN}-0.1 \% \mathrm{TFA} / 25 \mathrm{mM}$ diammonium citrate) and externally calibrated with a mixture of angiotensin $\mathrm{I}\left(5 \mathrm{pmol} \mu \mathrm{L}^{-1}\right)$-ACTH $\left(5 \mathrm{pmol} \mu \mathrm{L}^{-1}\right)$-insulin (100 pmol $\left.\mu \mathrm{L}^{-1}\right)$-cytochrome $\mathrm{C}\left(100 \mathrm{pmol} \mu \mathrm{L}^{-1}\right)$.

\subsection{Quantum yield (QY) measurements}

The QY of a compound is defined as the fraction of molecules that emit a photon after direct excitation by the source. ${ }^{38}$ This quantity is not the same as the total number of emitted photons which escape a bulk sample divided by the total number of absorbed photons, although in many instances the two quantities are nearly equal. The measurement of QY employed the comparison method which is described below (eqn (1)):

$$
\Phi_{\text {unk }}=\left(A_{\text {std }} / A_{\text {unk }}\right)\left(F_{\text {unk }} / F_{\text {std }}\right)\left(n_{\text {unk }}^{2} / n_{\text {std }}^{2}\right) \Phi_{\text {std }}
$$

where $\Phi=$ quantum yield; unk = unknown sample; std $=$ standard; $n=$ refractive index of solvent; $A=$ absorption at the selected excitation wavelength; $F=$ integrated fluorescence signal in the emission region. To calculate the QY of Au NCs, we 
measured a series of the samples and the standard 9,10diphenylanthracene $\left(\Phi=0.91\right.$ in ethanol) ${ }^{39}$ All the samples were diluted to ensure optical densities less than 0.02 measured by a Lambda 35 UV-visible spectrophotometer (Perkin-Elmer, USA) in order to reduce the error. The emission spectra were recorded on a FP-6500 fluorescence spectrometer (Jasco, JPN) under excitation of $375 \mathrm{~nm}$ light at $25^{\circ} \mathrm{C}$.

\section{Results and discussion}

\subsection{Synthesis and characterization of tridentate polymer ligands}

Three types of thioether polymer ligands PTMP-PMMA, PTMPPBMA and PTMP-P $t$ BMA were prepared by a facile chaintransfer radical polymerization method using pentaerythritol tetrakis-3-mercaptopropionate (PTMP) as chain-transfer agent, methyl methacrylate (MMA), $n$-butyl methacrylate (BMA) and tert-butyl methacrylate ( $t \mathrm{BMA})$ as monomers, respectively (Scheme 1a). The molecular weights and structures of polymer ligands were confirmed by GPC and ${ }^{1} \mathrm{H}$ NMR analyses (Fig. S1S4 and Table S1, ESI $\dagger$ ). None of the polymer ligands showed fluorescence under ultraviolet light and no emission spectra were observed (Fig. S5, ESI + ).

\subsection{Synthesis of blue emitting highly-fluorescent Au NCs}

Au NCs were prepared by irradiating a THF solution of $\mathrm{HAuCl}_{4}$ in the presence of the polymer ligand with $365 \mathrm{~nm}$ light (Scheme 1b). As shown in Fig. 1, three as-prepared Au NCs samples Au NCs@PTMP-PMMA, Au NCs@PTMP-PBMA and Au NCs@PTMP-P $t$ BMA exhibited the same fluorescence profile with two emission peaks at 412 and $433 \mathrm{~nm}$ and with excitation
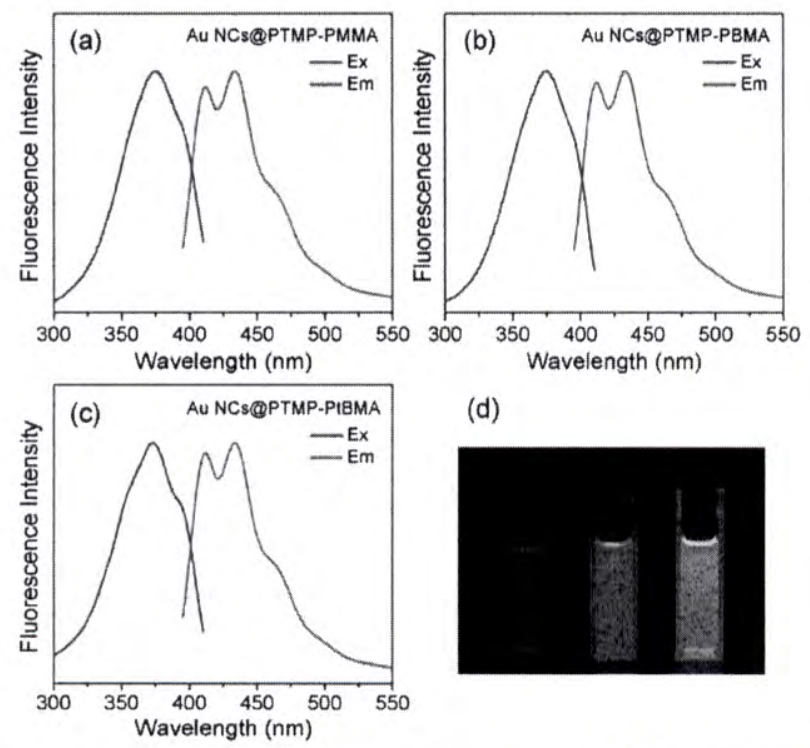

(d)

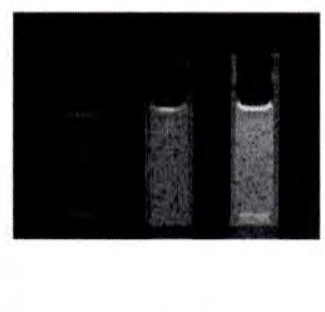

Fig. 1 Fluorescence emission and excitation spectra of (a) Au NCs@PTMPPMMA, (b) Au NCs@PTMP-PBMA and (c) Au NCs@PTMP-PtBMA. All the samples were dissolved in THF. (d) Photographs of Au NCs@PTMP-PMMA, Au NCs@PTMPPBMA and Au NCs@PTMP-PtBMA (from left to right) under irradiation of 365 nm UV light. maxima of $375 \mathrm{~nm}$. Their double emission peaks could be attributed to the surface properties of Au NCs influenced by the solvent, which lead to the electronic energy splitting and electron redistribution of the cluster surfaces. ${ }^{19}$ These Au NCs were well dispersed in THF and formed a colorless suspension in ambient light, while showing blue fluorescence under a UV lamp (Fig. 1d). The samples were kept at $-5{ }^{\circ} \mathrm{C}$ and their fluorescence could be retained for more than two months. The highest fluorescence quantum yields (QY) of Au NCs in THF solutions were measured to be $3.8,14.3$ and $20.1 \%$ for $\mathrm{Au}$ NCs@PTMP-PMMA, Au NCs@PTMP-PBMA and Au NCs@PTMP-P $t$ BMA, respectively.

The effect of the molar ratio between $\mathrm{Au}^{3+}$ ions and polymer ligands on the fluorescence of Au NCs was also investigated (Fig. S6, ESI $\dagger$ ). The optimum concentration of polymer ligands in order to obtain the brightest fluorescence was found to be $4 \mathrm{mM}$, which was the same for all the three polymer ligands. The variation in polymer concentration has little effect on the emission peak position of resulting Au NCs, but very low polymer concentration $(<1 \mathrm{mM})$ led to non-fluorescent species. This may be due to the insufficient polymer ligands which might not be able to effectively protect the Au NCs. When the polymer ligand concentration was increased from 4 to $8 \mathrm{mM}$, the dramatic decrease in the emission intensity can be attributed to limitation of the growth of Au NCs caused by excessive polymer ligands.

\subsection{Fluorescence mechanism with the evolution of Au NCs}

Fluorescence emission and absorption of the Au NCs were also studied at different UV irradiation times in order to explore the relationship between fluorescence properties and the growth of Au NCs. Before irradiation, none of the Au-precursor solutions showed fluorescence (Fig. 2a-c) and only the $\mathrm{Au}^{3+}$ ion absorption peak was found at $340 \mathrm{~nm}$ (Fig. 3). The initial Au solutions gradually changed from light yellow to colorless in $60 \mathrm{~min}$ accompanied with the evolution of emission peaks. A gradual increase in fluorescence intensity of Au NCs and a decrease in $\mathrm{Au}^{3+}$ ions absorption intensity were observed along with the increase in irradiation time. This indicates the observed fluorescence originates from small $\mathrm{Au}$ NCs formed by the reduction of $\mathrm{Au}^{3+}$ ions by $\mathrm{UV}$ light. ${ }^{40,41}$ The maximum fluorescence intensity appeared at $480 \mathrm{~min}$ with no absorption in the wavelength range from 400 to $700 \mathrm{~nm}$. DLS studies indicated the diameter of as-prepared Au NCs were ultra-small (Fig. S7a-c, ESI + ).

Prolonged irradiation led to a decrease in the fluorescence intensity with a visible color change in Au NCs solution from colorless into light pink (Fig. S8, ESI $\dagger$ ) and the characteristic surface plasmon resonance (SPR) peak of Au nanoparticles appeared at $540 \mathrm{~nm}$ in the absorption spectra when the irradiation time was extended to $600 \mathrm{~min}$. For Au NCs@PTMP-P $t$ BMA, the particle size was measured to be $2.3 \pm 0.1 \mathrm{~nm}$ by DLS (Fig. S7d, ESI $\dagger$ ), which also supports the observation of the SPR absorption band. TEM images further confirmed these changes, particles became irregular and polydispersed, their diameter increased to more than $2.0 \mathrm{~nm}$ and even larger (Fig. S9a, ESI $\dagger$ ). These drastic changes indicate that the Au NCs were aggregated 

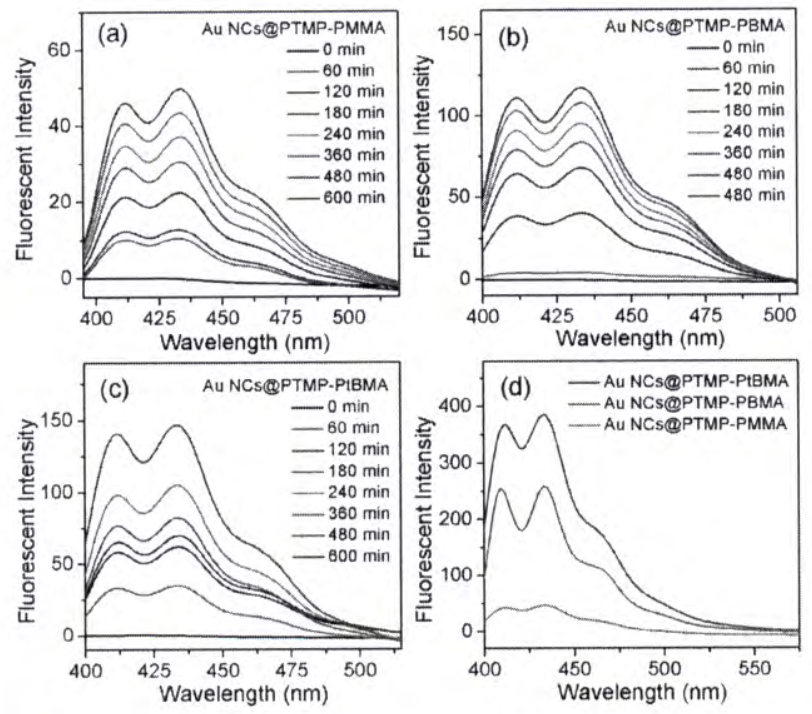

Fig. 2 Fluorescence emission spectra (excited at $375 \mathrm{~nm}$ ) of Au NCs prepared by different ligands (a) PTMP-PMMA, (b) PTMP-PBMA and (c) PTMP-PtBMA at different times under equivalent irradiation conditions. (d) Fluorescence emission spectrum of Au NCs using different polymer ligands at the same mole ratio and under the same irradiation conditions.
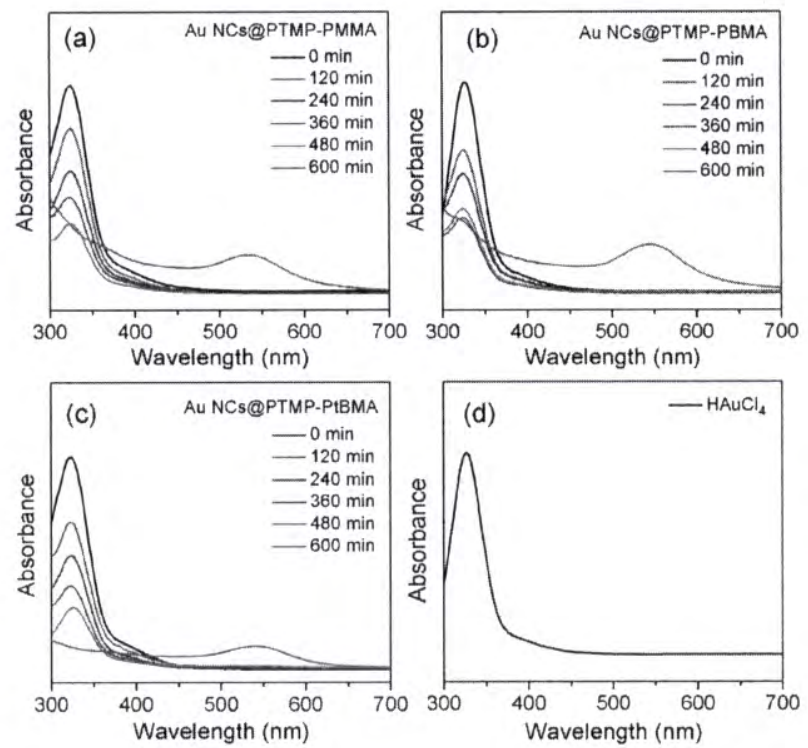

Fig.3 Time dependent evolution of UV-Vis spectra of (a) Au NCs@PTMP-PMMA, (b) Au NCs@PTMP-PBMA, (c) Au NCs@PTMP-PtBMA and (d) $\mathrm{HAuCl}_{4}$ in THF solution.

into Au nanoparticles in this process and the fluorescence of asprepared Au NCs is decreased. Furthermore, the NCs solution became darker in color gradually and turned into violet in one week at room temperature, which indicated these samples irradiated for a long time were not very stable and underwent slow aggregation. Interestingly, some irregular shapes of $\mathrm{Au}$ such as planar nanoplates more than $20 \mathrm{~nm}$ long were observed in TEM images (Fig. S9b, ESI + ), which may be due to the
Ostwald ripening process. ${ }^{42}$ The proposed synthesis and evolution of Au NCs is schematically summarized in Scheme 2a.

Zheng and co-workers suggested that these fluorescent $\mathrm{Au}$ NCs may be a complex composed of $\mathrm{Au}(0)$ atoms and a large percentage of $\mathrm{Au}(\mathrm{I})$ ions, and the fluorescence is associated with the fraction of $\mathrm{Au}(\mathrm{I})$ ions in the clusters. ${ }^{43} \mathrm{X}$-Ray photoelectron spectroscopy (XPS) measurements were carried out to analyse the variation of gold valence states in our Au NCs. The Au sample obtained from $480 \mathrm{~min}$ irradiation showed that the binding energies (BE) of $\mathrm{Au} 4 \mathrm{f}_{7 / 2}$ and $\mathrm{Au}_{4 \mathrm{f}_{5 / 2}}$ are 84.6 and $88.3 \mathrm{eV}$, respectively (Fig. $4 \mathrm{~b}$, solid line). It is notable that the $\mathrm{BE}$ of $\mathrm{Au} 4 \mathrm{f}_{7 / 2}$ falls in between the $\mathrm{Au}(0) \mathrm{BE}(84 \mathrm{eV})$ of a metallic gold film and the $\mathrm{Au}(\mathrm{I}) \mathrm{BE}(85 \mathrm{eV})$ of gold thiolate,${ }^{43,44}$ suggesting the co-existence of $\mathrm{Au}(0)$ and $\mathrm{Au}(\mathrm{I})$ in the clusters. ${ }^{45,46}$ The deconvolution of the $A u 4 f_{7 / 2}$ band revealed an asymmetric band that can be fitted into two peaks at 84.1 and $85.0 \mathrm{eV}$, which were assigned to $\mathrm{Au}(0)$ and $\mathrm{Au}(\mathrm{I})$, respectively (Fig. 4a). According to the integrated area ratio between these two peaks, nearly $60 \%$ of $\mathrm{Au}$ atoms in the $\mathrm{Au}$ NCs were $\mathrm{Au}(\mathrm{I})$ and significantly influence the $\mathrm{BE}$ of $\mathrm{Au}$ atoms. When the UV irradiation time was extended to $600 \mathrm{~min}$, the $\mathrm{BE}$ of $\mathrm{Au} 4 \mathrm{f}_{7 / 2}$ was shifted from 84.6 to $84.4 \mathrm{eV}$ (Fig. 4b), indicating the decrease in $\mathrm{Au}(\mathrm{I})$ fraction. These results support that the fluorescence from Au NCs is also associated with the presence of a fraction of $\mathrm{Au}(\mathrm{I})$ ions in the clusters. ${ }^{43}$

\subsection{The effect of polymer ligand structures on the fluorescence of Au NCs}

According to Jin and $\mathrm{Wu},{ }^{29}$ fluorescence of Au NCs could be largely facilitated by employing small molecular ligands with electron-rich atoms or groups. Since polymer ligands have longer carbon chains as compared to the small molecular ligands and small differences in molecular structure of the monomer would be greatly amplified after polymerization, introduction of high electron-donating capability could lead to a dramatic influence on the fluorescence properties of Au NCs. We assumed the Au NCs stabilized with PTMP-PtBMA would show the highest fluorescence intensity while those prepared with PTMP-PMMA would show the lowest. In order to prove this assumption, PTMP-PMMA, PTMP-PBMA and PTMP-P $t$ BMA were designed to have similar molecular weight by adjusting the concentration of chain transfer agent. The chain numbers (repeating units) of polymer ligands were estimated at 38, 37 and 36 for PTMP-PMMA, PTMP-PBMA and PTMP-PtBMA, respectively. Au NCs samples stabilized by these three different polymer ligands were prepared using the same ligand molar ratio $(4 \mathrm{mM})$ under identical irradiation conditions. The fluorescence intensity lies in the sequence Au NCs@PTMP-P $t$ BMA > Au NCs@PTMP-PBMA > Au NCs@PTMP-PMMA (Fig. 2d), which is consistent with the QYs mentioned before, confirming our speculation. Control experiments were performed by using a mixture of monomers (PMMA, PBMA and PtBMA) and PTMP instead of polymers as ligands. However, no fluorescent species could be observed. This indicates polymers play an important role in the obtaining of fluorescent Au NCs.

Scheme $2 \mathrm{~b}$ shows the possible interactions on the surface of $\mathrm{Au}$ NCs with tridentate polymer ligands PTMP-PMMA, 

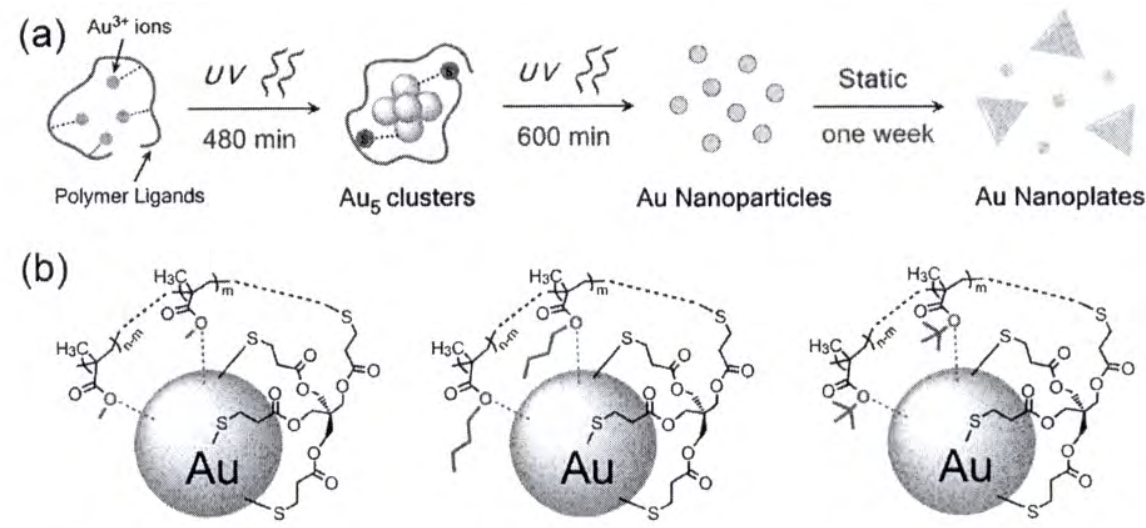

Scheme 2 (a) Schematic illustration of evolution of Au NCs. (b) Possible interactions of tridentate polymer ligands PTMP-PMMA, PTMP-PBMA and PTMP-PtBMA (from left to right) to the Au NCs surface. For clarity, only two repeat units are shown and not drawn to scale.
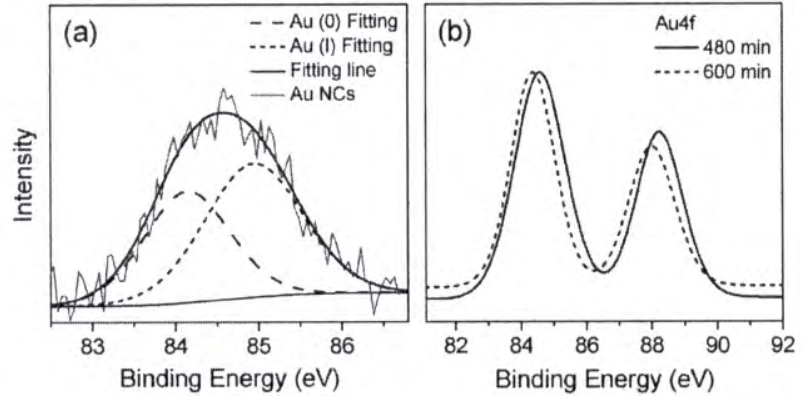

Fig. 4 XPS spectra showing the binding energy of Au NCs@PTMP-PtBMA. (a) $\mathrm{Au}_{4 / 2}$ was fitted considering two bands at 84.1 and $85.0 \mathrm{eV}$, which were assigned to $A u(0)$ and $A u(1)$, respectively. (b) Comparison of the $A u 4 f$ level of $A u$ NCs@PTMP-PtBMA irradiated for 480 and $600 \mathrm{~min}$

PTMP-PBMA and PTMP-PtBMA. Oxygen atoms from repeating units (e.g. $t \mathrm{BMA}$ in PTMP-PtBMA) donate delocalized electron density to the Au core while thiol groups of ligands can be attached to the Au core via strong S-Au bonds and stabilize the $\mathrm{Au}$ NCs. When the excited electrons are returned from the high energy state to the low energy states, they can transfer from ligands to the Au cores, which leads to an increase in fluorescence emission. ${ }^{29}$ Therefore, the capability of the polymer ligand to donate electrons plays an important role in improving fluorescence. The higher fluorescence intensity and QY of $\mathrm{Au}$ NCs@PTMP-P $t$ BMA and Au NCs@PTMP-PBMA than Au NCs@PTMP-PMMA could be attributed to the stronger electrondonating abilities of $t \mathrm{BMA}$ and BMA owing to their longer carbon chains. For Au NCs@PTMP-PtBMA and Au NCs@PTMPPBMA, the differences arise due to the isomeric structures of $t$ BMA and BMA. The tert-butyl group shows a hyper-conjugation effect, leading to stronger electron-donating abilities than the $n$-butyl group. Meanwhile, greater steric hindrance effect of surface PTMP-PtBMA may be protects the Au core against quenching by solvent. Both effects could result in the highest fluorescence intensity and QY of Au NCs@PTMP-PtBMA. On the basis of these results, we obtained highly fluorescent Au NCs by employing polymer ligands with high electron-donating ability and steric hindrance effect.

It should be noted that all these Au NCs exhibited the same fluorescence emissions at 412 and $433 \mathrm{~nm}$, similar to that of pepsin-mediated $\mathrm{Au}_{5} \mathrm{NCs}^{22}$ The fluorescence of metal NCs mainly depend on the particle size effect and the surface ligand effect. ${ }^{1}$ In order to determine the core size, we performed matrix-assisted laser desorption ionization mass spectrometry (MALDI-TOF) analysis (Fig. 5). The mass peak at $m / z=1081$ is assigned to the $\mathrm{Au}$ clusters with a composition of $\mathrm{Au}_{5} \mathrm{~S}_{3}$, whereas the other peaks could be assigned to the fragments of $\mathrm{Au}_{4} \mathrm{~S}_{3}(m / z=884), \mathrm{Au}_{3} \mathrm{~S}_{3}(\mathrm{~m} / z=687)$ and $\mathrm{Au}_{3} \mathrm{~S}_{2}(\mathrm{~m} / z=655)$. No larger $\mathrm{Au}_{n}$ clusters were found in higher mass range up to $\mathrm{m} / \mathrm{z}$ 10000 . MALDI-TOF results confirmed that all of Au NCs@ PTMP-PMMA, Au NCs@PTMP-P $t$ BMA and Au NCs@PTMPPBMA clusters have the same formulae and are composed of 5 gold atoms. Based on these results, for our $\mathrm{Au}_{5} \mathrm{NCs}$, surface

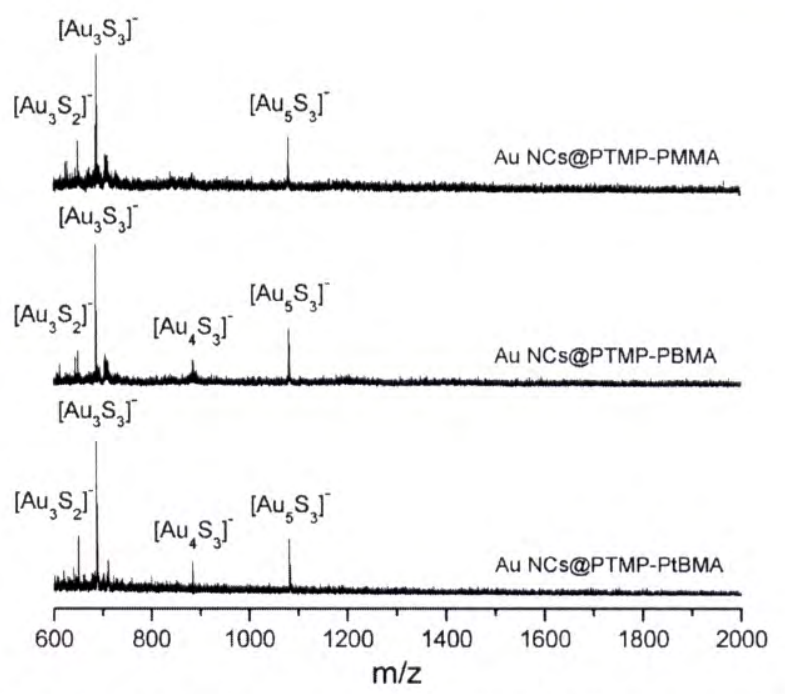

Fig. 5 MALDI-TOF mass spectra of Au NCs@PTMP-PMMA, Au NCs@PTMPPBMA and Au NCs@PTMP-PtBMA (from top to bottom) using CHCA as matrix. 
ligands have little influence on the excitation and emission profile but only result in different QYs, indicating the fluorescence wavelength dominated by core size of Au NCs, following a free-electron (Jellium) model. ${ }^{6}$

\subsection{The influence of temperature on the fluorescence of $\mathrm{Au}$} NCs

The influence of temperature on the fluorescence of Au NCs was investigated by recording fluorescence emission spectra at different temperatures in the range from 5 to $35{ }^{\circ} \mathrm{C}$ (Fig. S10, $\mathrm{ESI}+$ ). The fluorescence intensity was increased linearly with decreasing temperature, the relative fluorescence intensity being decreased from $100 \%$ at $5{ }^{\circ} \mathrm{C}$ to $90 \%$ at $35{ }^{\circ} \mathrm{C}$ (Fig. $\mathrm{S} 10$ inset, ESI $\dagger$ ). This could be explained as due to reduction in medium viscosity due to increase in temperature, which in turn leads to faster molecular movement rate and increased collision probability between the fluorescent Au NCs and solvent or other molecules and resulting in fluorescence quenching and reduction of QY. ${ }^{47}$ In addition, the fluorescence intensity decreased by $0.33 \%$ per degree centigrade, indicating that as-prepared $\mathrm{Au}$ NCs were only slightly sensitive to temperature. ${ }^{48}$

\section{Conclusions}

A simple and facile synthesis of highly-emissive blue fluorescent $\mathrm{Au}_{5}$ NCs is reported with non-fluorescence multidentate polymer ligands by photoreduction and the maximum QY of Au NCs was found to be $20.1 \%$. The fluorescence properties of Au NCs under UV irradiation was studied by optical spectra, XPS and MALDI-TOF, suggesting the fluorescence of Au NCs is sizedependent and is associated with the fraction of $\mathrm{Au}(\mathrm{I})$ ions in the clusters. Our work also demonstrates that the polymer ligand structure has a greater influence on QY than on emission of $\mathrm{Au}$ NCs. Polymer ligands with higher electron-donating abilities and steric hindrance effect could largely promote fluorescence, which might help in the synthesis and theoretical research of fluorescent metal NCs in the future.

\section{Acknowledgements}

We thank the Analysis and Testing Center, Huazhong University of Science and Technology for their assistance in characterization of materials. This work was financially supported by the National Natural Science Foundation of China (no. 51173058).

\section{Notes and references}

1 J. Zheng, C. Zhou, M. Yu and J. Liu, Nanoscale, 2012, 4, 40734083.

2 L. Shang, S. Dong and G. U. Nienhaus, Nano Today, 2011, 6, 401-418.

3 H. Xu and K. S. Suslick, Adv. Mater., 2010, 22, 1078-1082.

4 R. Jin, Nanoscale, 2010, 2, 343-362.

5 W. Wei, Y. Lu, W. Chen and S. Chen, J. Am. Chem. Soc., 2011, 133, 2060-2203.

6 J. Zheng and P. R. Nicovich, Annu. Rev. Phys. Chem., 2007, 58, 409-431.
7 L. Shang, L. Yang, F. Stockmar, R. Popescu, V. Trouillet, M. Bruns, D. Gerthsen and G. U. Nienhaus, Nanoscale, 2012, 4, 4155-4160.

8 X. Huang, Y. Luo, Z. Li, B. Li, H. Zhang, L. Li, I. Majeed, P. Zou and B. Tan, J. Phys. Chem. C, 2011, 115, 16753-16763.

9 R. Jin, H. Qian, Z. Wu, Y. Zhu, M. Zhu, A. Mohanty and N. Garg, J. Phys. Chem. Lett., 2010, 1, 2903-2910.

10 Z. Wu, C. Gayathri, R. R. Gil and R. Jin, J. Am. Chem. Soc., 2009, 131, 6535-6542.

11 B. Bellina, I. Compagnon, F. Bertorelle, M. Broyer, R. Antoine, P. Dugourd, L. Gell, A. Kulesza and V. Mitric, J. Phys. Chem. C, 2011, 115, 24549-24554.

12 T. Udaya Bhaskara Rao and T. Pradeep, Angew. Chem., Int. Ed., 2010, 49, 3925-3929.

13 Z. Wu, E. Lanni, W. Chen, M. E. Bier, D. Ly and R. Jin, J. Am. Chem. Soc., 2009, 131, 16672-16674.

14 L. Shang and S. Dong, Chem. Commun., 2008, 1088-1090.

15 X. Huang, B. Li, L. Li, H. Zhang, I. Majeed, I. Hussain and B. Tan, J. Phys. Chem. C, 2012, 116, 448-455.

16 N. Schaeffer, B. Tan, C. Dickinson, M. J. Rosseinsky, A. Laromaine, D. W. McComb, M. M. Stevens, Y. Wang, L. Petit, C. Barentin, D. G. Spiller, A. I. Cooper and R. Levy, Chem. Commun., 2008, 3986-3988.

17 J. Zheng and R. M. Dickson, J. Am. Chem. Soc., 2002, 124, 13982-13983.

18 J. Zheng, J. T. Petty and R. M. Dickson, J. Am. Chem. Soc., 2003, 125, 7780-7781.

19 R. Zhou, M. Shi, X. Chen, M. Wang and H. Chen, Chem.-Eur. J., 2009, 15, 4944-4951.

20 C. I. Richards, S. Choi, J.-C. Hsiang, Y. Antoku, T. Vosch, A. Bongiorno, Y.-L. Tzeng and R. M. Dickson, J. Am. Chem. Soc., 2008, 130, 5038-5039.

21 S. A. Patel, C. I. Richards, J.-C. Hsiang and R. M. Dickson, J. Am. Chem. Soc., 2008, 130, 11602-11603.

22 H. Kawasaki, K. Hamaguchi, I. Osaka and R. Arakawa, Adv. Funct. Mater., 2011, 21, 3508-3515.

23 K. Chaudhari, P. L. Xavier and T. Pradeep, ACS Nano, 2011, 5, 8816-8827.

24 H. Yabu, Chem. Commun., 2011, 47, 1196-1197.

25 H. Zhang, X. Huang, L. Li, G. Zhang, I. Hussain, Z. Li and B. Tan, Chem. Commun., 2012, 48, 567.

26 S. Liu, F. Lu and J.-J. Zhu, Chem. Commun., 2011, 47, 26612663.

27 L. Yan, Y. Cai, B. Zheng, H. Yuan, Y. Guo, D. Xiao and M. M. F. Choi, J. Mater. Chem., 2012, 22, 1000.

28 X. Yuan, Z. Luo, Q. Zhang, X. Zhang, Y. Zheng, J. Y. Lee and J. Xie, ACS Nano, 2011, 5, 8800-8808.

29 Z. Wu and R. Jin, Nano Lett., 2010, 10, 2568-2573.

30 C. M. Cobley, J. Chen, E. C. Cho, L. V. Wang and Y. Xia, Chem. Soc. Rev., 2011, 40, 44-56.

31 C.-A. J. Lin, T.-Y. Yang, C.-H. Lee, S. H. Huang, R. A. Sperling, M. Zanella, J. K. Li, J.-L. Shen, H.-H. Wang, H.-I. Yeh, W. J. Parak and W. H. Chang, ACS Nano, 2009, 3, 395-401.

32 I. Díez, H. Jiang and R. H. A. Ras, ChemPhysChem, 2010, 11, 3100-3104.

33 H. Duan and S. Nie, J. Am. Chem. Soc., 2007, 129, 24122413. 
34 Z. Wang, W. Cai and J. Sui, ChemPhysChem, 2009, 10, 20122015.

35 X. Huang, B. Li, H. Zhang, I. Hussain, L. Liang and B. Tan, Nanoscale, 2011, 3, 1600-1607.

36 Z. Wang, B. Tan, I. Hussain, N. Schaeffer, M. F. Wyatt, M. Brust and A. I. Cooper, Langmuir, 2007, 23, 885-895.

37 I. Hussain, S. Graham, Z. Wang, B. Tan, D. C. Sherrington, S. P. Rannard, A. I. Cooper and M. Brust, J. Am. Chem. Soc., 2005, 127, 16398-16399.

38 G. A. Crosby and J. N. Demas, J. Phys. Chem., 1971, 75, 9911024 .

39 M. Mardelli and J. Olmsted III, J. Photochem., 1977, 7, 277285.

40 K. G. Stamplecoskie and J. C. Scaiano, J. Am. Chem. Soc., 2011, 133, 3913-3920.
41 J. C. Scaiano, C. Aliaga, S. Maguire and D. Wang, J. Phys. Chem. B, 2006, 110, 12856-12859.

42 P. W. Voorhees, J. Stat. Phys., 1985, 38, 231-252.

43 C. Zhou, C. Sun, M. Yu, Y. Qin, J. Wang, M. Kim and J. Zheng, J. Phys. Chem. C, 2010, 114, 7727-7732.

44 M. A. Habeeb Muhammed, P. K. Verma, S. K. Pal, A. Retnakumari, M. Koyakutty, S. Nair and T. Pradeep, Chem.-Eur. J., 2010, 16, 10103-10112.

45 L. Shang, R. M. Dörlich, S. Brandholt, R. Schneider, V. Trouillet, M. Bruns, D. Gerthsen and G. U. Nienhaus, Nanoscale, 2011, 3, 2009-2014.

46 R. L. Whetten and R. C. Price, Science, 2007, 318, 407-408.

47 W. R. Ware and B. A. Baldwin, J. Chem. Phys., 1965, 43, 11941197.

48 R. F. Kubin and A. N. Fletcher, J. Lumin., 1982, 27, 455-462. 